\section{Averaging: An empirical validity criterion for magnitude estimation*}

\author{
DAVID J. WEISS \\ California State University, Los Angeles, California 90032
}

Ss judged grayness of neutral value Munsell chips under two response conditions: magnitude estimation and graphic rating. In addition, they judged average grayness of pairs of chips under the same two response modes. The averaging data were evaluated in terms of a simple model for subjective averaging. The graphic rating data fitted the model, but the magnitude estimates showed consistent discrepancy. It was concluded that the $\mathrm{Ss}$ were averaging, but that magnitude estimation distorted their judgments.

The method of magnitude estimation has had great appeal to those concerned with practical scaling problems, partly because of its simplicity and partly because of the regularity with which a power function describes the data. However, face validity is not a sufficient basis upon which to build a measuring instrument. Moreover, magnitude estimation data often disagree with those obtained from other response modes of equal simplicity (Stevens \& Galanter, 1957).

There are no compelling a priori grounds for choosing among response modes that yield different scales. Various writers (Torgerson, 1960; Galanter \& Messick, 1961; Schneider \& Lane, 1963) have labored to reconcile the differences on theoretical grounds. In contrast, the present research employs an empirical evaluation. A criterion for validity is established to which each response mode may be subjected.

The procedure rests upon the use of an information integration task, namely, averaging of grayness. Performance of this averaging task is evaluated in terms of a simple model for the integrative process. If the data from one response mode fit the model, while the data from another mode do not, then the failing mode is charged with invalidity. The assumption made here is that the data from the mode that supports the model provide evidence that the descriptive model is appropriate; the failing response mode distorts the judgments.

A constant-weight averaging model has been successful in describing the results of similar psychophysical averaging tasks (Anderson, 1967; Weiss

*This work was supported in part by National Science Foundation Grant GB-6666. The author wishes to thank Carlyn Joergensen for her assistance in running $S s$ and Michael $H$. Birnbaum for helpful comments on the manuscript. The author is especially grateful to Norman $H$. Anderson for guidance during all stages of this work.
\& Anderson, 1969). For the averaging of two graynesses, the model may be written as:

$$
\begin{aligned}
\mathbf{R}_{\mathbf{i j}}= & \mathbf{w}_{\mathrm{L} \psi}\left(\mathbf{S}_{\mathbf{i}}\right)+\mathbf{w}_{\mathbf{R} \psi\left(\mathbf{S}_{\mathrm{j}}\right)} \\
& \mathbf{w}_{\mathbf{L}}+\mathbf{w}_{\mathbf{R}}=1
\end{aligned}
$$

$\mathbf{R}_{\mathbf{i j}}$ is the response to the stimulus pair, $S_{i}$ (on the left) and $S_{j}$ (on the right). $\psi$ represents the psychophysical rather than stimulus values that are combined in the judgment. The weights $w_{L}$ and $w_{R}$ are assumed to depend only upon physical position.

The averaging model is tested directly upon the raw data. If the stimulus pairs are constructed according to a factorial design, then the averaging model makes a simple parallelism prediction. Acceptance of this model allows interval scales to be constructed from the data, using functional measurement procedures (Anderson, 1970). Single-stimulus judgments were also obtained to provide a comparison with these constructed scales.

\section{METHOD}

\section{Task Definition}

Magnitude estimation. The $\mathbf{S}$ was told to judge the grayness (or average grayness) of the stimulus (or stimulus pair) in relation to that of the standard (or standard pair), which was to be called 100. Darker stimuli were to be given higher numbers. The standard was present throughout the magnitude estimation phases of the experiment. The instructions stressed the incorporated practice in the use of numbers to express given ratios.

Graphic rating. Judgments were expressed by making a slash mark on a 137-mm line. The line was drawn $19 \mathrm{~mm}$ below the top of a $72 \mathrm{x}$ $137 \mathrm{~mm}$ strip of paper. Scale-defining stimuli (or stimulus pairs) were displayed throughout this condition. The S was told that the grayness (or average grayness) of the lighter function, as it is perceived values importance of expressing ratios and scale-defining stimulus was less than any of the other stimuli used; it was to correspond to a point "about here" on the response line (the $E$ pointed to a spot about $10 \mathrm{~mm}$ from the left end of the line). The darker scale-defining stimulus defined the right end of the line in a similar way.

\section{Design}

The pairs to be averaged were constructed according to $a$ by 5 factorial design, with the factors corresponding to the left and right spatial positions as the pair was presented. Stimulus values will be given in Munsell values rather than in reflectances. The levels for the left factor were, from light to dark: $9,7.5$, $6,4.5,3$; the levels for the right factor were: $8,6.5,5,3.5,2$. For magnitude estimation, the standard pair was $(6$, 4.5); the pairs of scale-defining stimuli for graphic rating were $(9.5,9)$ and $2.5,2)$. For single-stimulus judgments, the standard was 5.5 and the scale-defining stimuli were 9.5 and 2 .

\section{Procedure}

Each of eight paid Ss was run individually through five daily sessions of about $50 \mathrm{~min}$ each. The same order of conditions was followed for each $\mathrm{S}$. Magnitude estimation was used on Days 1 and 5 and graphic ratings on Day 3 . The second and fourth sessions were devoted to similar judgments of angle averaging, which have been reported in a methodological paper (Weiss \& Anderson, 1972). Each session consisted of four replications of single-stimulus judgments and four replications of averaging judgments. Instructions were read before the first replication every day.

\section{Stimuli}

The stimuli were Munsell neutral value chips, $5 / 8 \times 7 / 8$ in, matte finish. Each chip was mounted on a clear plastic staff that was inserted in a small vial filled with clay. When presented for judgment, the vial was placed in a stand behind a brown panel in such a way that only the chip and a $25 \cdot \mathrm{mm}$ segment of the staff were visible.

Two stands were used. For the graphic rating stand, two pairs of scale-defining stimuli flanked the stimuli to be judged. In the magnitude estimation stand, the standard sitmuli were $175 \mathrm{~mm}$ to the left of the stimuli to be judged. For the single-stimulus judgments, there was only one pair of scale-defining stimuli and one standard stimulus.

\section{RESULTS}

Averaging Model

Graphic test. The data for grayness averaging are shown in Fig. 1. The 


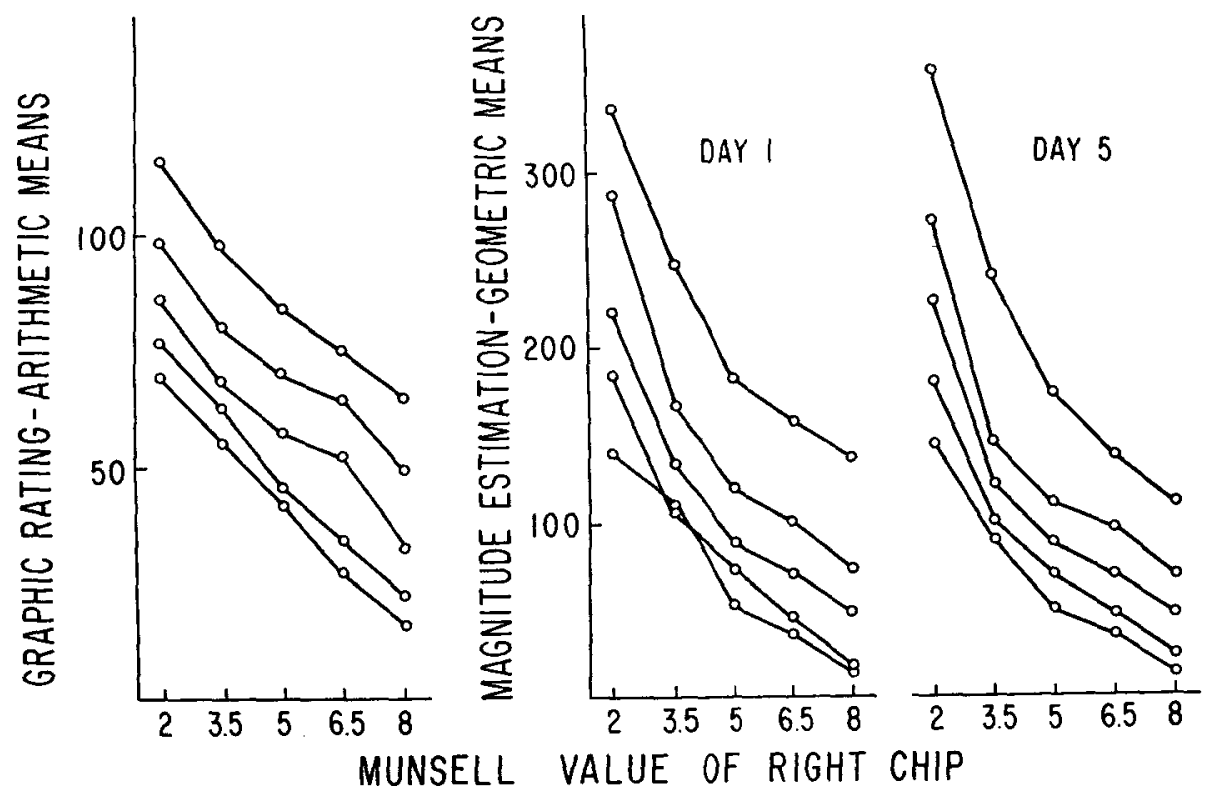

Fig. 1. Mean responses for grayness averaging task: arithmetic means of graphic ratings in left panel, geometric means of magnitude estimations in right panels. Each curve corresponds to one value of the left chip.

additive model of Eq. 1 implies that the lines within each panel should be parallel.

The graphic ratings in the left panel appear to verify this parallelism prediction. That means that the stimulus factors do combine in an additive manner; that, in turn, supports the model, which asserts that Ss do average grayness.

The magnitude estimation data are distinctly nonparallel, showing a strong divergence at the dark end of the continuum. Geometric means have been plotted, but the graphs are virtually identical for arithmetic means. Comparison of the two panels suggests that no important changes took place over the course of the experiment; however, it may be worth noting that the Day 5 data are more regular. The nonparallelism means that an additive model is not appropriate. The magnitude estimation graphs show that the response may not be described as an arithmetic mean.

Statistical test. The additive model may also be tested statistically. The model implies that the 5 by 5 factorial design should produce no interaction; in practice, the interaction should be no nsignificant. Accordingly, group analyses of variance were applied to the responses for each condition. In such a group analysis, the appropriate error term for an effect is its interaction with Ss.

For practical and theoretical reasons, it was decided to decompose the interaction into a bilinear component and a remainder in carrying out the significance tests. The bilinear component nor the remainder were significant, using the pooled error term. The $F$ ratios were 2.35 ( $\mathrm{df}=$ $1,112$ ) and 1.70 (df $=15,112)$. These statistical tests thus support the additive model for graphic ratings.

For both sets of magnitude estimation data, the bilinear component was large and highly significant (Day 1: $\mathrm{F}=16.12$, $\mathrm{df}=$ 1,112; Day $5: F=49.18$, $\mathrm{df}=1,112$ ), while the $F$ ratios for the remainders were less than 1 . These tests mean that the additive model does not hold for the magnitude estimations.

This bilinear analysis is consistent with the idea that the magnitude estimations have a multiplicative form. That, in turn, supports the above suggestion that the bias in magnitude estimation is an exponential transformation. This suggestion is consistent with the well-documented finding that category scales appear to be logarithmic functions of magnitude scales for various continua (Stevens \& Galanter, 1957).

\section{Functional Scales}

Acceptance of the averaging model for the graphic rating data follows the construction of two interval scales. These constructed grayness scales are simply the marginal means of the factorial design; the scale values are linearly related to $\psi\left(\mathrm{S}_{\mathbf{i}}\right)$ and $\psi\left(\mathrm{S}_{\mathrm{j}}\right)$ (Anderson, 1970, Eq. 2). As the scales are unique only up to a linear transformation, the highest and lowest constructed grayness values for each scale have been set equal to their objective reflectance values.

Figure 2 shows that the functional scale is slightly concave downward when plotted against reflectance. Figure 3 shows that the functional scale is approximately the Munsell scale, although the functional scale is slightly more positively accelerated.

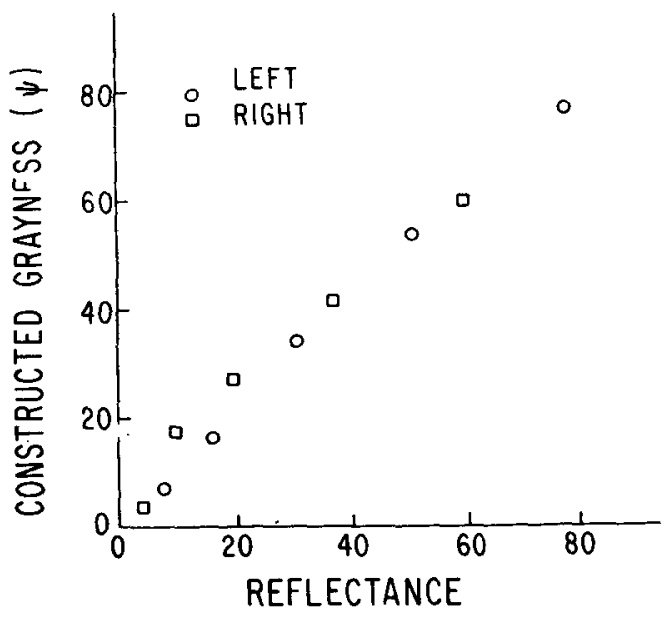

Fig. 2. Functional scales based on graphic ratings, plotted against reflectance, 
Single-Stimulus Judgments

The graphic ratings of the single stimuli are shown in Fig. 4. Plotted against Munsell value, the mean responses appear to follow a resonably straight line.

The magnitude estimates are shown in Fig. 5. Plotted on logarithmic coordinates, the data clearly do not conform to the straight line that a power function requires; rather, the curves are well described as line segments joined in the region of the standard (see Poulton, 1968). These data thus replicate those of Torgerson (1960) for magnitude estimates of darkness of Munsell chips.

\section{DISCUSSION}

The data in Fig. 1 cast serious doubt upon the validity of magnitude estimation as a response techuique. The graphic rating data confirm that the Ss were able to average graynesses; magnitude estimation produced a bias in their responses.

This result highlights the advantage of using an integration task to provide integration process must be the same in both response modes. When one with the model and the other response mode yields data that disagree, the discrepancy can be attributed to the techniques are not merely discrepant; one is valid and the other is not.

It may be noted that the tests employed here are made directly upon the raw data; no assumption about the form of the psychophysical function is necessary. In functional measurement procedures, scales follow directly from the successful application of a judgmental model.

Some problems of magnitude a criterion for assessing validity. The response mode yields data that agree response mode. The response

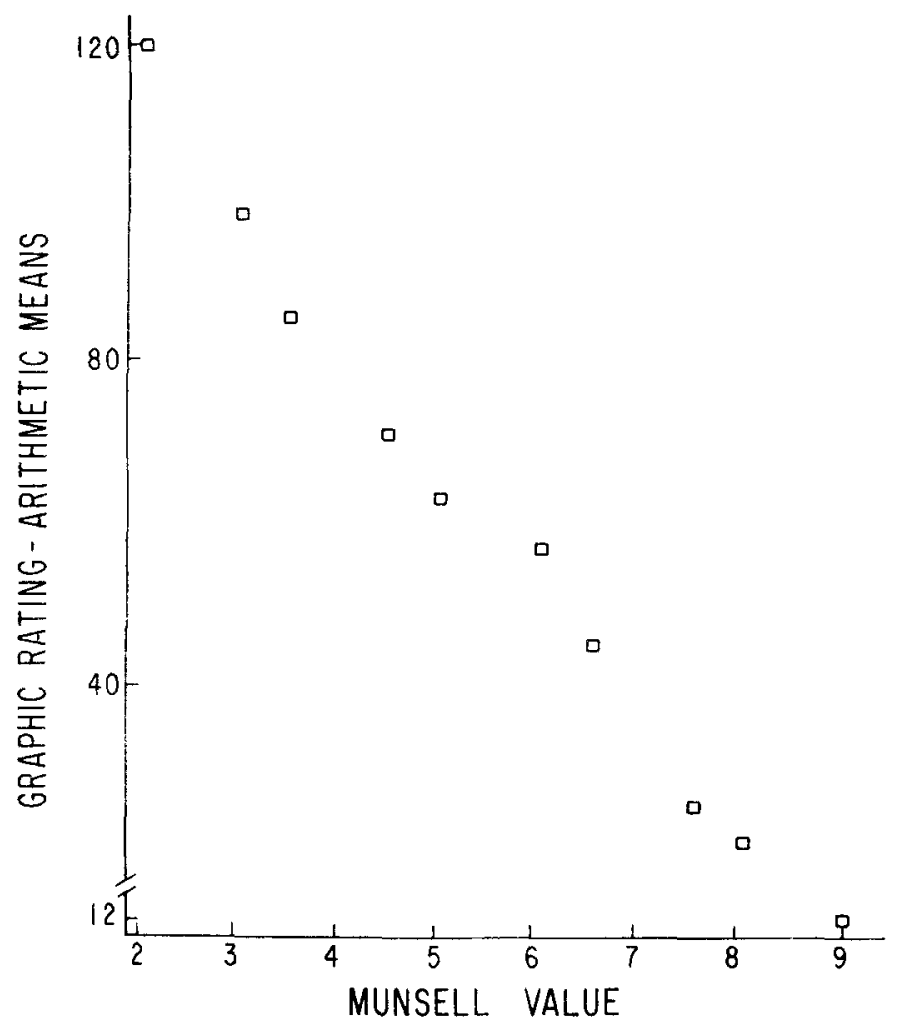

Fig. 4. Arithmetic means for graphic ratings of single chips, plotted against Munsell value.

estimation have been reported by other investigators who have used integration tasks. Dawson (1971) used magnitude estimation of apparent sums and differences of loudness stimuli, concluding that the resulting scales were not linearly related to the magnitude estimates of single stimuli. The most extensive work has been done by Curtis and his colleagues

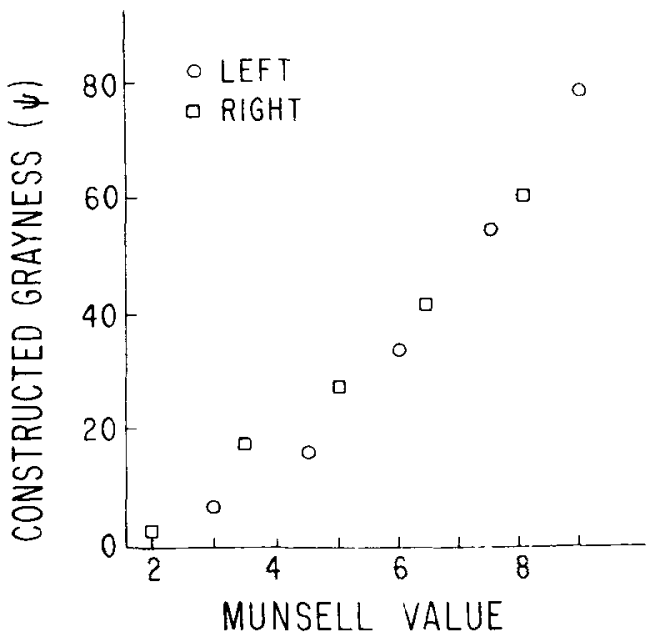

Fig. 3. Functional scales based on graphic ratings, plotted against Munsell value.
(Curtis, Attneave, \& Harrington, 1968; Curtis \& Fox, 1969; Curtis, 1970), who have used estimates of sums and differences of weights and brightness stimuli. Curtis (1970) concluded that magnitude estimation introduces a nonlinear output function.

This nonlinear bias appears to be a general but not universal rule. Weiss and Anderson (1972) found that graphic ratings of average inclination were more regular than magnitude estimates, but that an additive model fit both sets of data adequately. Miller and Sheldon (1969) reported that exponents for average length and average inclination correspond closely to those for length and inclination.

The present data do allow a logical possibility that magnitude estimates are not invalid. There is always a certain indeterminacy when a model is linked to a response system. If the Ss were taking geometric means of the stimulus pairs, then a multiplicative model would be appropriate. Such a model would, by virtue of the bilinear analysis, fit the magnitude estimates. Following this logic, the graphic ratings would be a logarithmic distortion. However, a geometric averaging model would not fit the inclination data of Weiss and Anderson (1972); there an arithmetic averaging model fit the data from both response 


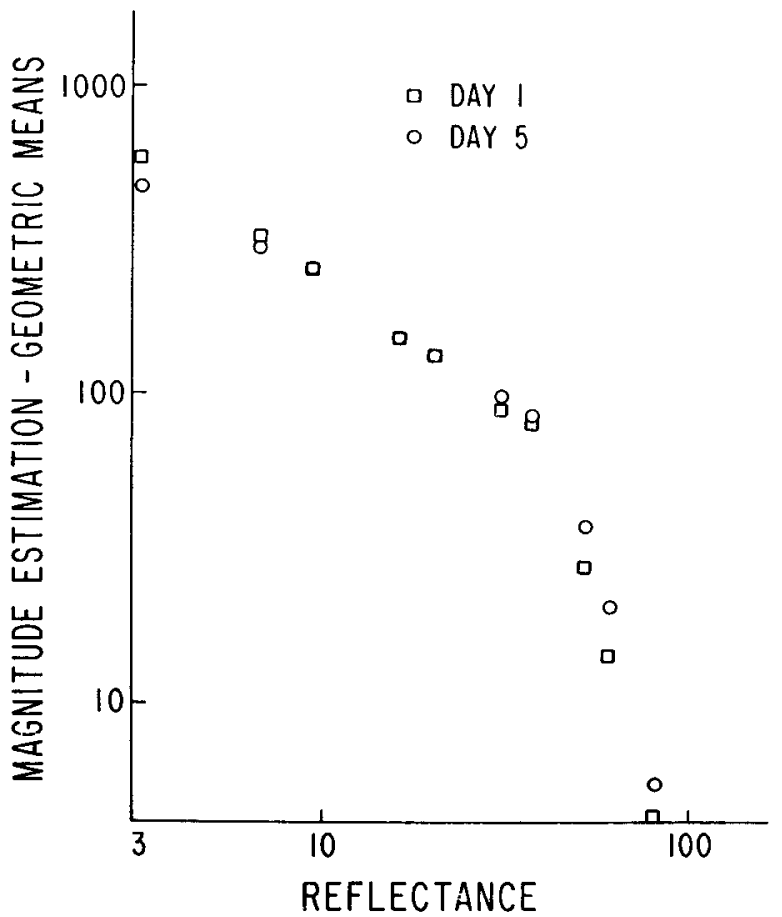

Fig. 5. Geometric means for magnitude estimations of single chips, plotted against reflectance. Log-log coordinates are used.

modes. Nevertheless, the ambiguity in any single experiment remains. This problem of interpretation is discussed in more detail by Anderson (1972).

Functional measurement approaches the validity issue by establishing psychological laws. Validity is conferred when an empirical criterion is satisfied, a criterion that does not depend on a priori assumptions about the form of the psychophysical function. When such a criterion is enforced, magnitude estimation fails to demonstrate its validity.

\section{REFERENCES}

ANDERSON, N. H. Application of a
Association, Portland, Oregon, April 1972.

CURTIS, D. W. Magnitude estimations and category judgments of brightness and brightness intervals: A two-stage interpretation. Journal of Experimental Psychology, 1970, 83, 201-208.

CURTIS, D. W., ATTNEAVE, F., \& HARRINGTON, T. L. A test of a two-stage model for magnitude estimation. Perception \& Psychophysics, $1968,3,25-31$.

CURTIS, D. W., \& FOX, B. E. Direct quantitative judgments of sums and a two-stage model for psychophysical judgments. Perception \& Psychophysics, $1969,5,89-93$.

DAWSON, W. E. Magnitude estimation of apparent sums and differences. Perception \& Psychophysics, 1971, 9, 368-374.

GALANTER, E., \& MESSICK, $S$. The relation between category and magnitude scales of loudness. Psychological Review, $1961,68,363-372$.

MILLER, A L. \& SHELDON R. Magnitude estimation of average length and average inclination. Journal of Experimental Psychology, 1969, 81, 16-21.

POULTON, E. C. The new psychophysics: Six models for magnitude estimation. Psychological Bulletin, 1968, 69, 1-19.

SCHNEIDER, B., \& LANE, H. Ratio scales, category scales, and variability in the production of loudness and softness. Journal of the Acoustical Society of America, 1963, 35, 1953-1961.

STEVENS, S. S. \& GALANTER, E. H. Ratio scales and category scales for a dozen perceptual continua. Journal of Experimental Psychology, 1957, 54, 377-411.

weighted average model to a psychophysical averaging task. Psychonomic Science, 1967, 8, 227-228. ANDERSON, N. H. Functional measurement and psychophysical judgment. Psychological Review, 1970, 77, 153-170.

ANDERSON, N. H. Cross-task validation of functional measurement. Perception \& Psychophysics, 1972, 12, 389-395.

ANDERSON, N. H., \& SHANTEAU, J. C. Information integration in risky decision making. Journal of Experimental Psychology, 1970, 84, 441-451.

ANDERSON, N. H., \& WEISS, D. J. Test of a multiplying model for estimated area of rectangles. American Journal of Psychology, 1971, 84, 543-548.

BIRNBAUM, M. H., \& VEIT, C. T. Information integration with difference, ratio, and averaging tasks. Paper presented at the Western Psychological
TORGERSON, w, $S$ Quantitative judgment scales. In $\dot{H}$. Gulliksen and $S$. Messick (Eds.), Psychological scaling. New York: Wiley, 1960.

WEISS, D. J \& ANDERSON N. H. Subjective averaging of length with serial presentation. Journal of Experimental Psychology, 1969, 82, 52-63.

WEISS, D. J., \& ANDERSON, N. H. Use of rank order data in functional measurement. Psychological Bulletin, $1972,78,64-69$.

(Received for publication July 5, 1972.) 